\title{
Prerequisites for the Establishment of the Auto- mated Monitoring System and Accounting of the Displacement of the Roof of Underground Mines for the Improvement of Safety of Mining Work
}

\author{
Alexandr Abramovich ${ }^{1 *}$, Evgeniy Pudov ${ }^{1}$, and Evgeny Kuzin ${ }^{1}$ \\ ${ }^{1}$ T.F. Gorbachev Kuzbass State Technical University, 653039, Nogradskaya str., 19a, Prokopievsk, \\ Russia
}

\begin{abstract}
In the article the necessity of continuous control over the condition of the roof of mine workings is considered, to increase the safety in the conduct of mining operations. Provided the rationale for monitoring in complex mining and geological conditions, as well as in areas prone to rock blows and sudden coal emissions. The existing methods for controlling the displacement of the roof rocks are described, and their shortcomings are given. An idea is given of an automated system for monitoring the displacement of the workings. The stages of the system as a whole are considered, including the choice of a linear displacement sensor, a platform for software development, and a programming language. In order to ensure integration into other systems and subsequent analysis of the results, it is envisaged to output data to spreadsheets. Are shown the interfaces of the program and the output of the readings from the sensors to the monitors of the mining manager.
\end{abstract}

\section{Introduction}

Increasing the safety of coal production remains an urgent task given the growing concentration of equipment and mining. One of the factors of possible accidents is the development of areas dangerous for mountain impacts and sudden emissions. In this case, the task is to determine the zone of destruction of the rock massif in the vicinity of the mine and the ends. The shape and size of the fracture zone depend on the actual stresses and strength of the rocks, on the depth of the workings, on the presence of geological disturbances.

Sudden geodynamic manifestations in the roof of the excavations are caused by a number of factors: geological disturbances of rocks, overmoistening of the massif, exceeding the load on the anchor support, simultaneous development of several layers and inadequate support for given geological conditions, these are just some of the many of these factors. A big step towards solving this problem is to identify the causes of collapse. Sometimes the causes of

* Corresponding author: abramovich_sanja@,rambler.ru 
roof collapse can not be determined, but the study of the fall of exfoliated and decomposed rocks, the state of the surrounding and geotechnical environment can reveal the causes of collapse [1].

An increasing amount of mined coal, an increase in the depth of excavations require improved methods for monitoring the condition of the roof, with high reliability of results, speed and continuity of control.

The Regulation on the Safe Management of Mining [2] regulates the conduct of research at each deposit, prone or dangerous for mining impacts, to improve the forecasting and prevention of mountain impacts. In this case, the forecast of the impact hazard of sections of the rock massif should be carried out, both according to the systems of continuous automated control of the stressed state of the array, and by changing the values (speeds) of deformations and displacements.

The variety of mining-geological and mining-technological factors continues to create complexity in predicting the manifestations of rock pressure.

In order to identify the patterns of rock pressure, timely detection of complex situations for managing rock pressure and crepe work, taking measures to eliminate the accident rate of mining operations, it is necessary to accumulate information and create a database with data on the magnitude and speed of displacement of the roof sections. Effective work is possible using a system of continuous monitoring of roof movements, analyzing the influence of various factors, and issuing information on dangerous sections of excavations, where it is necessary to take measures to strengthen the fastening.

\section{Materials and methods}

At present, methods of numerical simulation of geomechanical processes are being conducted, cores are selected, the structure of the roof rocks is analyzed using a video endoscope and GPR method $[3,4]$.

In addition, today, enterprises that mine coal underground, to control the displacements of rocks in the excavations used reference depth stations, which allow for visual contact to detect the displacement of rock mass. The method of visual control with the help of deep reference stations is to observe the rock shift by recording the readings when moving the frames fixed in the hole at the appropriate distance $[5,6]$.

Nowadays, this method is obsolete and has a number of shortcomings [7-9]:

1. For specific, clear information, it is necessary to monitor the frames continuously, that is, one directly needs to continuously record the shifts that occurred.

2. The uncertainty of the exact time of occurrence of the displacement of the rock mass, as well as the lack of information on the rate of its displacement.

Proceeding from the above, it becomes clear that the engineer and technical worker of the site, checking the state of the reference deep stations during the shift, with a rapid and large displacement of the roof rocks may not be in time to warn other employees of the mine. As a rule, this is done by the mining master once a shift, and it is not possible to say for what time the movement occurred.

The foreign researchers results [10-12], show the urgency of creating an automated monitoring system, with various ways of implementation.

Thus, our main task is to create a full-fledged automatic monitoring and accounting system for the displacement of the roof of underground workings, to improve the safety of mining, which will directly include:

1. Sensors of displacement;

2. Transmitting devices;

3. Communication channels;

4. Software; 
5. Output of data to the monitors of the mountain dispatcher.

The work on the creation itself will consist of several stages, mentioned earlier:

1. Select a displacement bias sensor.

2. Select the transmitter.

3. Determination of the method of data transmission (selection of data transmission channels).

4. Definition of the software development platform.

5. Software development.

The displacement sensor is a device designed to determine the magnitude of the linear or angular mechanical displacement of an object. It should be noted that all displacement sensors can be divided into two main categories - linear displacement sensors and angular displacement sensors (encoders). Of all the existing types of sensors, we are most suited to linear displacement sensors.

Having analyzed the merits and demerits of various converters, relative to the necessary parameters, it can be concluded that the most acceptable as a displacement sensor for the roof are capacitive linear displacement sensors with a variable plate area. The main advantages of capacitive converters are the simplicity of the device, high sensitivity, low power consumption, no moving contacts, comparative ease of manufacture, small dimensions and weight, long service life.

At the stage of approach to software development, we have constructed a general scheme for representing the system as a whole, a diagram (see Figure 1).

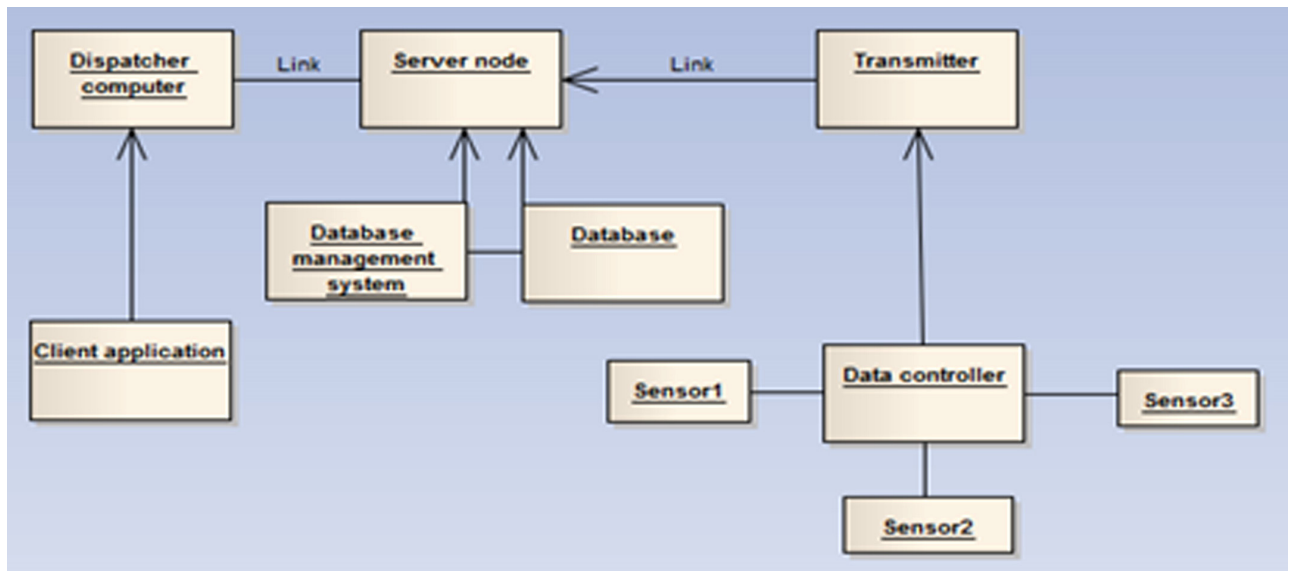

Fig. 1. System representation diagram.

\section{Results and discussion}

At the software development stage, the subject area was first analyzed. The most important point to which we drew attention is very large volumes of information, and it was decided to store all non-recurring recorded rock mass readings taken from each displacement sensor.

The architecture of the information system being developed is the client-server architecture. This architecture is implemented by the database management system (DBMS) of MSSQL Server.

Using this DBMS will provide the following functions:

- Ensures the integrity of the database (DB);

- provides fast recovery after various failures (hardware and software);

- provides backup;

- provides high operational reliability; 
- provides high performance.

During the development of the database it was decided to create a table for each sensor and thus the following information objects were identified:

- Sensor 1

- Sensor 2

- Sensor $n$

Integrity requirement: to table contents Sensor 1, Sensor 2, Sensor n must necessarily refer to level 1, level 2, date and time of reading, for two-level sensors. As a result, we got a rational database for storing information, a database diagram (see Fig. 2).

\begin{tabular}{|l|}
\hline Sensor1_Table \\
\hline Level1:double \\
\hline Level2:double \\
\hline Date_And Time: datetime \\
\hline
\end{tabular}

\begin{tabular}{|l|}
\hline Sensor2_Table \\
\hline Level1:double \\
\hline Level2:double \\
\hline Date_And Time: datetime \\
\hline
\end{tabular}

\begin{tabular}{|l|}
\hline SensorN_Table \\
\hline Level1:double \\
\hline Level2: double \\
\hline Date_And Time: datetime \\
\hline
\end{tabular}

Fig. 2. Resulting ER diagram (entity diagram-connection) of the database.

After implementing the data warehouse, we went on to the process of creating the program itself.

As technology of interaction of user-defined components with data, ADO.NET technology is chosen - the main model of data access for applications based on Microsoft .NET. The choice of technology is based on the choice of operating system. As a programming environ-

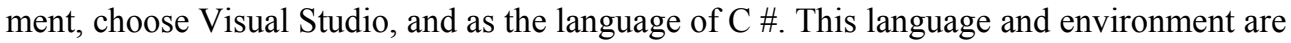
universal programming tools, so they are suitable for solving the set task of creating a system. The development of an implementation model (program code) can be represented as a description of the components that implement the boundary object classes and entity classes.

The implementation model includes the components integrated into the subsystems that implement the objects identified in the design process. The component model is shown in Fig. 3. 


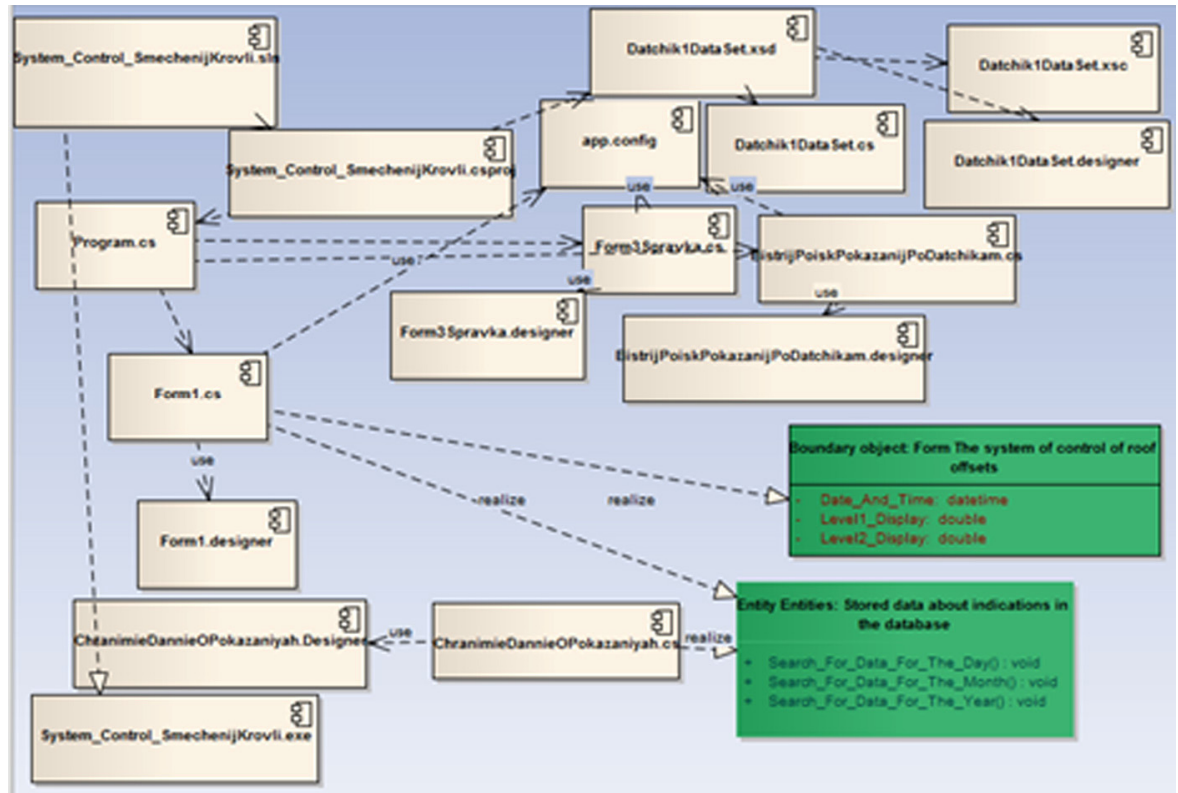

Fig. 3. Component model.

For each form and bookmark on the form, the composition of interactions with the user is determined, thanks to which the process of receiving and accounting of the readings of the sensors is carried out, as well as notification of the controller about the displacement of each sensor in a particular area.

To fulfill each functional requirement, we select according to a separate scenario. Scenario for fulfilling the functional requirement of finding the readings of the selected sensor for the entire time since the introduction of the system and installing the sensor:

The dispatcher has the ability to plot the selected sensor for the entire time, from the moment of the sensor introduction, as well as for the day, week, month, year.

To implement this scenario, two stored procedures were created in the database, which will be called in a program created on the Windows platform. As part of the system being developed, the client component and the server component are distinguished. The server component implements scenarios related to access to the data and its main processing. The client component implements the presentation of data in a user-friendly form and selects the actions to be performed.

The developed interface of the program is presented in Fig. 4

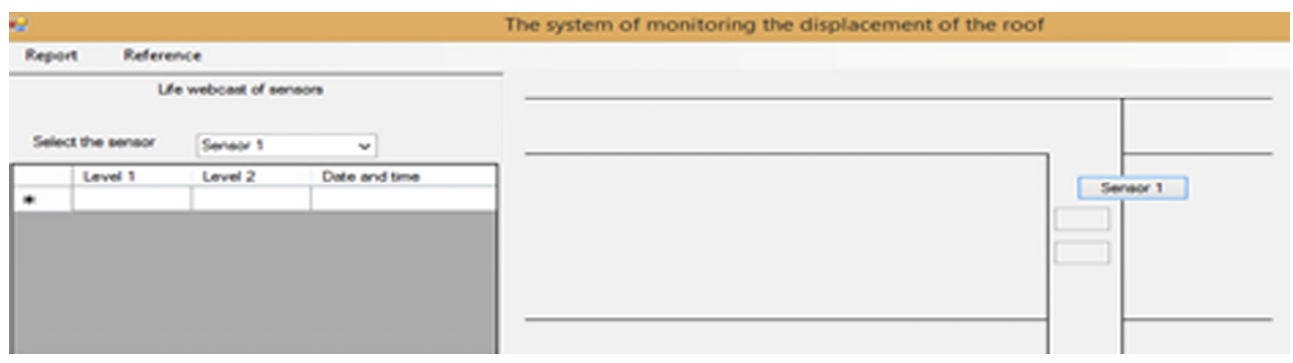

Fig. 4. Main page program interface.

The interface of the program for each separate sensor was also developed. The interface is shown in Fig. 5 below. 


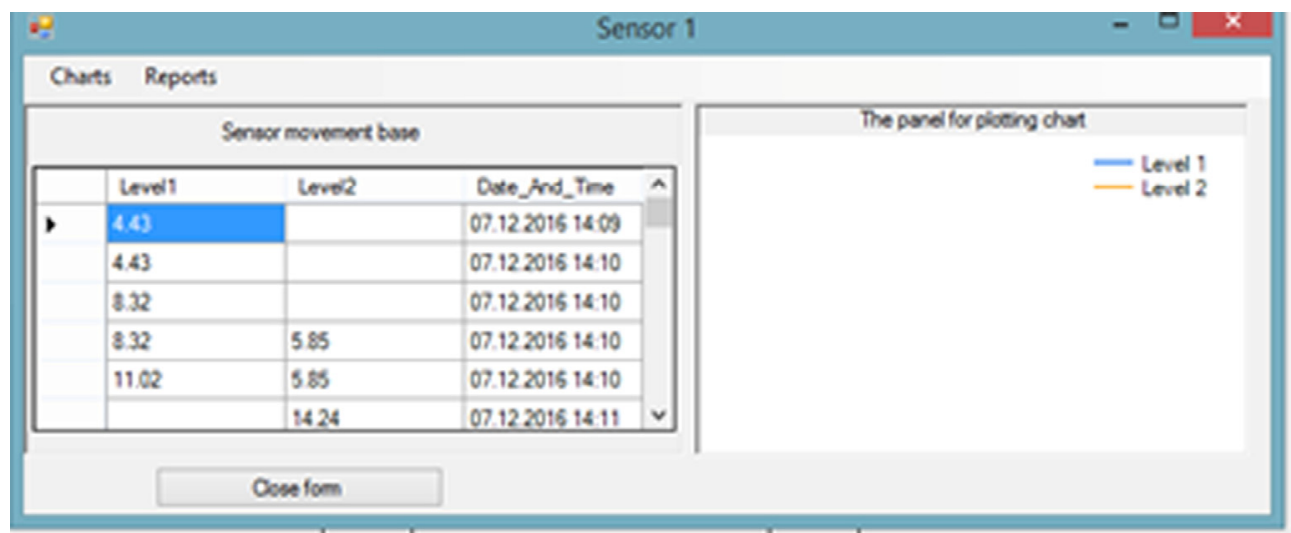

Fig. 5. Sensor 1 interface in the system for controlling the displacement of the mine workings.

In connection with the possible emergence of the need to work with the received data, including processing and analysis, it was decided to comprehensively export the received data to a Microsoft Office Excel document.

To implement the output of data in an Excel document, an Excel Report button was created on the form.

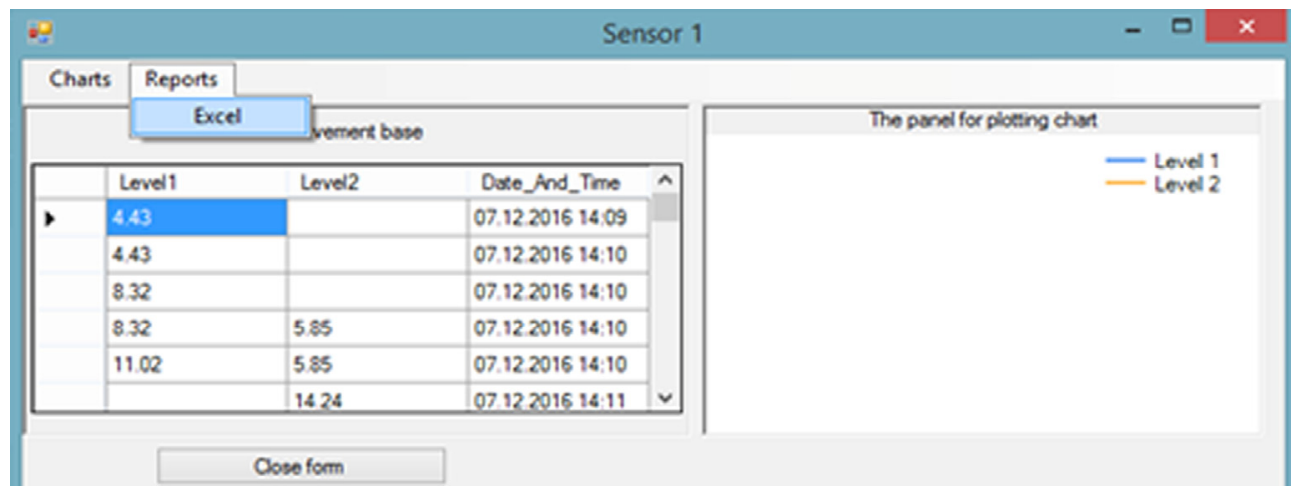

Fig. 6. Report interface in a spreadsheet.

\section{Conclusions}

As a result of the work, the first version of the system for monitoring and recording the displacement readings in the mine workings roof was created, which will allow to record changes in the state of the roof of underground mine workings in real time. Based on the accumulated information, it is possible to predict the behavior of the roof in such mining and geological conditions, and also to analyze the causes preceding the change in the roof state (displacement, stratification, decompression).

\section{References}

1. G. Molinda, C. Mark, Electron Journal Geotech Engineering, 15, 547 (2010) 
2. The order of Rostechnadzor from 02.12.2013 N 576 «About approval of Federal norms and rules in the field of industrial safety "Regulations for the safe conduct of mining works on deposits prone and dangerous mountain blows» (Registered in Ministry of justice of Russia, 2014).

3. G.L. Feofanov, Coal, 3, 18 (2017)

4. Haijun Zhao, Fengshan Ma, Jiamo Xu, Jie Guo, International Journal of Rock Mechanics \& Mining Sciences, 53, 20 (2012)

5. M.I. Alvarez-Fernandez, C. Gonzalez-Nicieza, A. Menendez-Diaz, Alvarez-Vigil., Eng. Geol., 80, 1 (2005)

6. Federal occupational safety standards and regulations «Guidelines for computation and application of coal mines rock support», (Moscow, JTC NTS Industrial Safety Publ., 2015).

7. Chunbin Zhao, Yubao Zhang, Genu Zhang, Janji Li, Shuki Ma., 17:5, 1044. (2017)

8. P. James Doherty, Allsize Hasan, H. Gonzalo Suazo, A. Andy Fury, Canadian Geotechnical Journal, 2:12, 1901 (2015)

9. E Lawson, T. Douglas, M. K. Larson, 35th International Conference on Ground Control in Mining (2016)

10. Yiming Zhao, Nong Zhang, Guangyao Si., Sensors (Basel), 16:10 (2016)

11. Z.Z. Zhang, J.B. Bai, Y. Chen, S. Yan, Rock Mech. Min. Sci., 80:1, (2015)

12. A. Catalano, F.A. Bruno, M. Pisco, A. Cutolo, A. Cusano, Sensors. 14, 18268 (2014) 
